\title{
Adherence to the Strengthening the Reporting of Observational Studies in Epidemiology (STROBE) checklist in articles published in EAACI Journals: a bibliographic study.
}

\author{
Elena Wiehn ${ }^{1}$, Cristian Ricci ${ }^{2}$, Aberto Alvarez-Perea ${ }^{3}$, Michael Perkin ${ }^{4}$, Christina Jones ${ }^{5}$, \\ Cezmi Akdis ${ }^{6}$, Jean Bousquet ${ }^{7}$, Philippe Eigenmann ${ }^{8}$, Clive E.H. Grattan ${ }^{9}$, Christian \\ Apfelbacher ${ }^{10}$, and Jon Genuneit ${ }^{1}$ \\ ${ }^{1}$ Ulm University \\ ${ }^{2}$ Leipzig University \\ ${ }^{3}$ Hospital General Universitario Gregorio Maranon \\ ${ }^{4}$ St George's, University of London \\ ${ }^{5}$ University of Surrey Faculty of Health and Medical Sciences \\ ${ }^{6}$ University of Zurich \\ ${ }^{7}$ University Hospital \\ ${ }^{8}$ University Hospital Geneva, Switzerland \\ ${ }^{9}$ Guy's Hospital \\ ${ }^{10}$ Universität Magdeburg Medizinische Fakultät
}

March 12, 2021

\begin{abstract}
Research data derived from observational studies are accumulating quickly in the field of allergy and immunology and a large amount of observational studies are published every year. The aim of the present study was to evaluate the adherence to the Strengthening the Reporting of Observational Studies in Epidemiology (STROBE) checklist by papers published in the three European Academy of Allergy and Clinical Immunology journals, during the period 2009-2018. To this end, we conducted a bibliographic study of up to eight randomly selected papers per year per Journal. Our literature search resulted in 223 papers. Among those, 80, 80 and 63 records were from Pediatric Allergy and Immunology, Allergy and Clinical and Translational Allergy, respectively; the latter was published only from 2011 on. Prospective, case-control, and cross-sectional designs were described in 88, 43, and 92 papers, respectively. Full reporting of all STROBE items was present in $47.4 \%, 45.6 \%$, and $41.2 \%$ for the cohort, cross-sectional, and case-control studies, respectively. Generally, no time trend in adherence of reporting STROBE items was observed, apart from reporting funding, which increased from $60 \%$ in 2009/2010 to more than $90 \%$ in 2018 . We identified a cluster of STROBE items with low proportions of full reporting constituted by the items on reporting study design in the title and methods, variables types along with their measurement/assessment, bias and confounding, study size, and grouping of variables. It appears that the STROBE checklist is a suitable tool in observational allergy epidemiology. However, adherence to the STROBE checklist appeared suboptimal.
\end{abstract}

Adherence to the Strengthening the Reporting of Observational Studies in Epidemiology (STROBE) checklist in articles published in EAACI Journals: a bibliographic study.

Running title: STROBE adherence in EAACI Journals.

Elena Wiehn ${ }^{{ }^{*}}$, Cristian Ricci ${ }^{2 *}$, Alberto Alvarez-Perea ${ }^{3}$, Michael R. Perkin ${ }^{4}$, Christina J. Jones ${ }^{5}$, 
Cezmi Akdis ${ }^{6}$, Jean Bousquet ${ }^{7}$, Philipe Eigenmann ${ }^{8}$, Clive Grattan ${ }^{9}$, Christian J. Apfelbacher ${ }^{10,11}$, Jon Genuneit ${ }^{1,2}$, for the Task Force "Adherence to reporting guidelines in articles published in EAACI Journals: a systematic review." of the EAACI Working Group on Epidemiology

${ }^{1}$ Institute of Epidemiology and Medical Biometry, Ulm University, Ulm, Germany

${ }^{2}$ Pediatric Epidemiology, Department of Pediatrics, University Medicine Leipzig, Leipzig, Germany

${ }^{3}$ Allergy Department, Hospital General Universitario Gregorio Marañón, Madrid, Spain

${ }^{4}$ Population Health Research Institute, St George's, University of London, London, United Kingdom

${ }^{5}$ Faculty of Health \& Medical Sciences, School of Psychology, University of Surrey, Guildford,

United Kingdom

${ }^{6}$ Swiss Institute of Allergy and Asthma Research, University of Zurich, Davos, Switzerland

${ }^{7}$ University Hospital, Montpellier, France

${ }^{8}$ University Hospital Pediatrics, Geneva, Switzerland

${ }^{9}$ St John's Institute of Dermatology, Guy's Hospital, London, United Kingdom

${ }^{10}$ Institute of Epidemiology and Preventive Medicine, University of Regensburg, Regensburg, Germany

11 Institute of Social Medicine and Health Systems Research, Otto von Guericke University Magdeburg, Magdeburg, Germany

* Authors contributed equally to the manuscript

\section{Abstract (248 words)}

Research data derived from observational studies are accumulating quickly in the field of allergy and immunology and a large amount of observational studies are published every year. The aim of the present study was to evaluate the adherence to the Strengthening the Reporting of Observational Studies in Epidemiology (STROBE) checklist by papers published in the three European Academy of Allergy and Clinical Immunology journals, during the period 2009-2018. To this end, we conducted a bibliographic study of up to eight randomly selected papers per year per Journal. Our literature search resulted in 223 papers. Among those, 80, 80 and 63 records were from Pediatric Allergy and Immunology, Allergy and Clinical and Translational Allergy, respectively; the latter was published only from 2011 on. Prospective, case-control, and cross-sectional designs were described in 88, 43, and 92 papers, respectively. Full reporting of all STROBE items was present in $47.4 \%, 45.6 \%$, and $41.2 \%$ for the cohort, cross-sectional, and case-control studies, respectively. Generally, no time trend in adherence of reporting STROBE items was observed, apart from reporting funding, which increased from $60 \%$ in 2009/2010 to more than $90 \%$ in 2018 . We identified a cluster of STROBE items with low proportions of full reporting constituted by the items on reporting study design in the title and methods, variables types along with their measurement/assessment, bias and confounding, study size, and grouping of variables. It appears that the STROBE checklist is a suitable tool in observational allergy epidemiology. However, adherence to the STROBE checklist appeared suboptimal.

\section{Introduction}

Allergic diseases represent a public health issue ${ }^{1}$. Up to $30 \%$ of individuals may be affected by some type of allergic diseases whose prevalence, as a whole, is on the rise ${ }^{2-5}$. Scientific evidence from observational studies is accumulating quickly in the field of allergy epidemiology, as also indicated by the increasing number of systematic reviews in allergy epidemiology ${ }^{6,7}$. However, the availability of a vast number of published papers 
does not necessarily correspond to the timely translation of such research into public health and prevention programmes. This is also true in allergy epidemiology, where delivery of care and healthcare services have not evolved sufficiently despite an increased prevalence of allergic diseases ${ }^{8}$. Evidence-based prevention has become a topic of great interest in the field of allergy immunology and its implementation is clearly strategic ${ }^{9}$.

The quality of reporting of allergy observational studies is crucial for any kind of subsequent research and utilization, including the translation into effective and efficient public health and evidence based prevention programmes ${ }^{10,11}$. Peer-reviewed journals in the field of allergy epidemiology require that papers from observational studies have a certain quality of reporting. Tools have been proposed to guide or evaluate the quality of reporting in observational studies. Those tools are collected by the EQUATOR network ${ }^{12}$. The Strengthening the Reporting of Observational Studies in Epidemiology (STROBE) is one of the most comprehensive tools in use. STROBE is also widely accepted and recommended by the International Committee of Medical Journal Editors https://www.strobe-statement.org/index.php?id=strobe-endorsement. After a three-year research process involving more than 35 scientists, the STROBE checklist was first published in 2007. It consists of 22 items, four of which are specifically defined by study design (cross-sectional, cohort and case-control) while the other 18 items evaluate certain aspects of reporting quality that are shared by all observational designs ${ }^{13}$. The STROBE checklist is also attractive because it evaluates reporting quality by paper section with specific items for title and abstract, introduction, methods, results and discussion. Thus, STROBE appears to be a suitable tool to evaluate reporting quality in observational research; however, its correct use is not free from pitfalls ${ }^{14}$. STROBE is of qualitative nature so its quantitative use is challenging. For example, it is not intuitive to understand if and how, following STROBE assessment, the reporting from a given study can be considered as poor or satisfactory from an overall or section-specific viewpoint.

This study was developed and conducted by a Task Force "Adherence to reporting guidelines in articles published in EAACI Journals" of the European Academy of Allergy and Clinical Immunology (EAACI). The aim of this study was to evaluate the degree to which the 22 STROBE items were adhered to in research published in the field of allergy and whether this varied over time and by study design.

\section{Methods}

We ran a MEDLINE literature search of observational studies conducted in humans and published in one of the three European Academy of Allergy and Clinical Immunology (EAACI) journals, Allergy, Clinical and Translational Allergy (CTA), and Pediatric Allergy and Immunology (PAI), between 2009 (2011 for CTA, its foundation year) and 2018. The literature search was conducted using the search string ("pediatr allergy immunol" [TA] OR "clin transl allergy"[TA] OR "allergy"[TA]) AND 2009:2018 [DP] NOT ("Editorial"[PT] OR "Letter" [PT] OR "Review" [PT] OR "Clinical Trial"[PT] OR "Case Reports"[PT])).

The search string generated overall 2,707 records with a median of 328 papers identified per year, ranging between 263 papers published in 2014 to 392 papers published in 2009. We randomized within journal and year. Only observational studies in humans to which STROBE would apply were eligible. So, we retained the first 8 eligible papers on that randomly ordered list. In case the full-text revealed that this was not an observational study, we excluded the paper and replaced it with the next paper on the random list. In the end, PAI contributed 80 records ( 8 per each of the 10 years; $35.9 \%$ ), Allergy contributed 80 records (35.9\%) and Clinical and Translational Allergy (CTA ) contributed 63 records $(28.2 \%)$ because it was only founded in 2011 with and had published only 9 suitable observational studies in its inaugural year.

\section{Data extraction, data collection and scores computation}

An evaluation of the data extraction procedure was performed to assess the agreement of two authors (EW and JG) on a subset of five randomly chosen papers. Subsequently, all data were extracted by one author (EW) using an electronic data capturing system developed using the Access 2016 desk application of Microsoft Office vers. 2010. Paper characteristics such as publication year, journal and study design along with the STROBE items scores were collected.

Several of the 22 STROBE items were further divided into components defined in the STROBE explanatory 
article ${ }^{15}$. For example, this article states for the $8^{\text {th }}$ STROBE item (method assessment): "For each variable of interest, give sources of data and details of methods of assessment(measurement). Describe comparability of assessment methods if there is more than one group". In this example, three specific sub-items in which the reporting of the features "sources of data", "methods of assessment" and "comparability of assessment" were coded separately. Details regarding the 22 STROBE items and their sub-items are reported in Supplementary table 1. A four level grading system was used for the STROBE items and sub-items: "fully reported", "not reported", "reported by citation", and "not applicable". "Reported by citation" indicates that the authors referred to a citation implicating that this contains the full reporting for that given feature; however, we did not verify if this was the case by reading the referenced paper.

Statistical analysis

We coded the 22 STROBE items as article section and article sub-section items attributing a dichotomous YES/NO score according to a conservative (all sub-items defined by the STROBE explanatory article were satisfied) and liberal (at least 50\% of sub-items were satisfied) criteria. Items without sub-items, i.e. the STROBE items on discussion and funding, were coded the same for the conservative and liberal score. Afterwards, the qualitative nature of STROBE was taken into account describing the 22 dichotomous scores by means of percentages. We merged results regarding multiple STROBE items belonging to the same article section by means of medians. Finally, we performed a multiple correspondence analysis, which is an analogue of principal component analysis applied to contingency tables from categorical data. We applied this technique to investigate the multivariate association between STROBE items and their correlation with study design. The correspondence analyses was performed using the FactoMineR package of the $\mathrm{R}$ software. Results are reported using graphical representations as bar charts of frequencies, scatter plot of percentages over publication years and correspondence analysis plot of the first two dimensions.

\section{Results}

There were 88 papers (39.5\%) with a prospective study design while $43(19.3 \%)$ and $92(41.2 \%)$ papers had a case-control and cross-sectional design, respectively. Amongst all included papers, $45.4 \%$ fully reported all 22 STROBE items according to our conservative definition or by citation. This differed by study type with $47.8 \%$ for cohort studies, $45.7 \%$ for cross-sectional studies, and $41.4 \%$ for case-control studies ( $\mathrm{p}_{\mathrm{ChiSq}}=0.007$ for the overall comparison of the three designs). This difference was brough about by the case-control studies which differed from cohort $\left(\mathrm{p}_{\mathrm{ChiSq}}=0.0017\right)$ and cross-sectional studies $\left(\mathrm{p}_{\mathrm{ChiSq}}=0.035\right)$; the difference between cohort and cross-sectional studies was not statistically significant. This difference by study design remains when using our liberal scoring of the $22 \mathrm{STROBE}$ items or reporting by citation. Here, we observed $67.0 \%$ for cohort studies, $64.1 \%$ for cross-sectional studies, and $58.1 \%$ for case-control studies $\left(\mathrm{p}_{\mathrm{ChiSq}}<0.0001\right.$ and $\mathrm{p}_{\mathrm{ChiSq}}=0.0015$ comparing case-control to cohort and to cross-sectional studies, respectively; $\mathrm{p}_{\text {ChiSq }}<0.0001$ for the overall comparison of the three designs; no statistically significant difference between cohort and cross-sectional studies).

A clear difference between our conservative and liberal scoring definitions to the STROBE checklist was observed for the title and abstract sections. This was due to the low proportion of papers reporting their study design in the title or abstract (28.3\%). Of note, this proportion was higher for cohort studies and crosssectional studies (36.4\% and $33.7 \%$, respectively) than for case-control studies (18.6\%). The percentages of full reporting by item, paper section, and study design are shown in figure 1.

The overall score was stable over time, irrespective of our conservative or liberal definition. Investigating sections of the STROBE, we observed that funding reporting increased from less than $60 \%$ to more than $90 \%$ while the scoring for other STROBE sections remained rather constant over the period from 2009 to 2018 (Fig 2).

Finally, results from the correspondence analyses show that its first two dimensions together explain $29.6 \%$ and $36.9 \%$ of the overall variance in our conservative and liberal STROBE scores, respectively. The first dimension separates full reporting from "not reported", explaining $18.6 \%$ and $23.9 \%$ of the overall variance in our conservative and liberal scores, respectively. The positioning of study designs along this dimension 
shows that reporting in papers based on a cohort study was more complete than reporting in papers based on a case-control design; here, the cross-sectional studies had an intermediate position. For the conservative score, the second dimension separated the STROBE items 1, 4, 7-11, 14, 17, and 21 from the other STROBE items. Notably, those items investigate reporting of features of the study design in the title and methods, of types of variables and their measurement/assessment, of bias and confounding, of study size, and of grouping of variables. These items are also the more often poorly reported ones. In the correspondence analysis of our liberal score, this cluster is extended to the items $6,9,10$, and 12 from the methods section, 16 and 19 from the results section, and item 22 regarding the funding source. In essence, the second dimension separates items from the methods and results sections with low proportion of full reporting from the other STROBE items. Again, positioning of the study designs along this dimension separates cohort and crosssectional designs from case-control studies confirming the aforementioned poor reporting in papers based on a case-control design.

\section{Discussion}

Adherence to the STROBE checklist has been previously investigated in other research fields ${ }^{16-19}$. Our observed proportions of full reporting are in line with those detected in other fields. This may suggest that the STROBE checklist is equably accepted and thus a suitable tool in observational allergy epidemiology. However, alike in other research fields, only a minority (45\%) of the allergy papers published in EAACI journals fully adhered to the reporting of all STROBE items.

We applied a rigorous random procedure to select the included papers to avoid potential selection bias. However, a limitation of our study is that we restricted our literature search to three journals affiliated with a strong European professional association. These are widely accepted in the field and ranked on positions 2,5 , and 8 (out of a total of 28 journals) in the category "Allergy" of the 2019 Journal Citation Reports@) (Clarivate Analytics). Suboptimal reporting quality in top journals in the field, despite a lag of 10 years since introduction of the STROBE checklist, is likely to reflect an issue generalizable to other journals in the field, also outside of Europe. One may be inclined to think that reporting quality may be even worse in lower ranking journals or journals not affiliated with a strong professional society. This would have to be explored by extending the present work to more journals in the field and some previous evidence argues against this assumption. A study of 69 studies, a mix of experimental and observational studies in animals and humans published in 2016, did not find a strong or statistically significant inverse correlation between reporting quality and the journal's impact factor ${ }^{20}$. Alike, a study of 171 observational studies affiliated with the Iranian Shiraz University of Medical Sciences did not show a correlation between STROBE-ascertained reporting quality and the journal's impact factor ${ }^{21}$.

The STROBE checklist was not designed to be used as a score or to rank papers by reporting quality after their publication, but as a checklist to be used when papers are written. We extended its use and demonstrated how to apply the STROBE checklist in a quantitative way, proposing conservative or liberal definitions. Using these proposed scores, we were able to identify differences in reporting quality by study design which could be viewed as external validation: papers based on cohort and cross-sectional designs had comparable reporting quality while papers based on case-control designs less often achieved high reporting quality. This has been found in other similar research as well but the underlying reasons remain elusive ${ }^{21}$. Of note, papers based on a case-control design were the minority among those we included in the present evaluation. This may reflect that there are several more methodological issues associated with case-control studies ${ }^{22-24}$ which may lead to the lesser use or publication of this study design.

Different sections of the papers achieved higher levels of full adherence to the STROBE criteria. In particular, high proportions of full reporting were observed for introduction, discussion, and funding. On the contrary, the reporting quality for the methods and results sections was lower and items in the methods and results sections with low levels of full reporting clustered together in the correspondence analyses. Specifically in the methods sections, we identified study settings, participant's features, efforts undertaken to account for bias and confounding, and sample size justification as the most unreported features. This poor reporting poses a trifold problem for translation of the reported evidence into public health policies and interventions. 
Firstly, study settings and participant's features must be well defined to identify the target populations. Secondly, residual bias or confounding limit applicability and efficacy. Thirdly, an undersized study comes with loss of statistical power potentially leading to false negative outcomes and thus potential discarding of valid ideas. Two STROBE items on sample description and reporting results from the main and secondary analyses (items 14 and 17) were particularly correlated to the aforementioned cluster of poorly reported items. Of note, full reporting within this cluster of poorly reported items was less achieved in case-control studies compared to cohort and cross-sectional studies, reinforcing the notion about poor reporting in papers based on case-control studies. Again, reporting of information on the target population as well as the results, including secondary and sensitivity analyses, which are the ones supposed to reveal potential source of biases, should be at the core of every scientific paper.

To improve the quality of reporting of studies, Moher et al. made four suggestions ${ }^{25}$. First, they proposed publication officers at universities and other research institutions, alike grants officers or technology transfer officers, who could provide guidance on manuscript preparation. Second, core competencies for editors should be established including knowledge on reporting guidelines. Third, training authors in scientific writing and adherence to reporting guidelines. Fourth, peer reviewers could receive formal training, e.g. at universities.

In addition to structural implementation of officers and training programs at research institutions, interventions at the journal level have been suggested. The obligatory submission of filled reporting guideline checklists (STROBE, CONSORT, PRISMA) along with manuscript submission raised the adherence to these checklists by $13 \%$ for observational studies to $58 \%$ for systematic reviews ${ }^{26}$. A suggestion derived from this study was to implement checklists in online submission systems, which can be ticked by authors as well as the reviewers ${ }^{26}$. Furthermore, a scoping review found 31 interventions to improve reporting but only 4 were tested in randomised trials ${ }^{27}$. The mere endorsement of the use of reporting guidelines by a scientific journal influenced the reporting quality little or not at $\mathrm{all}^{27}$. Improvements were achieved by active interventions on editors and peer reviewers, who were required to assess adherence to the appropriate checklist ${ }^{27}$. Even though some of the interventions were evaluated and proven to be effective, they are still not realized in the routine of scientific work ${ }^{27}$.

All three evaluated EAACI journals endorse the use of reporting guidelines including STROBE in their author guidelines. Active, structural implementation of reporting guidelines in the submission process, as well as, during editorial and peer review evaluation including training for editors and peer reviewers seems warranted but will require larger efforts. Until this is achieved, we suggest to start with simple, targeted interventions based on our results. For instance, authors could be required to select their study design from a list in the submission system. This may be used to instruct editors or peer reviewers to evaluate the two or three most poorly reported STROBE items for that given study design. It may also be used to append a generic subtitle to all published manuscripts disclosing the study design. We hope that our present work provides basis for improving reporting quality of observational studies in allergy research, both by initiation of targeted interventions on journal level as well as by increased awareness among authors.

\section{Author Contribution}

All authors contributed to the design of the study. EW, CR, AAP, CJJ and JG contributed to article selection and data extraction. EW, CR and JG conducted the analyses and wrote the initial draft of the manuscript. All authors contributed to the interpretation of the data, revised the manuscript and approved the final version.

Figure 1. STROBE checklist response rates by paper section and study design using a conservative or liberal scoring. 

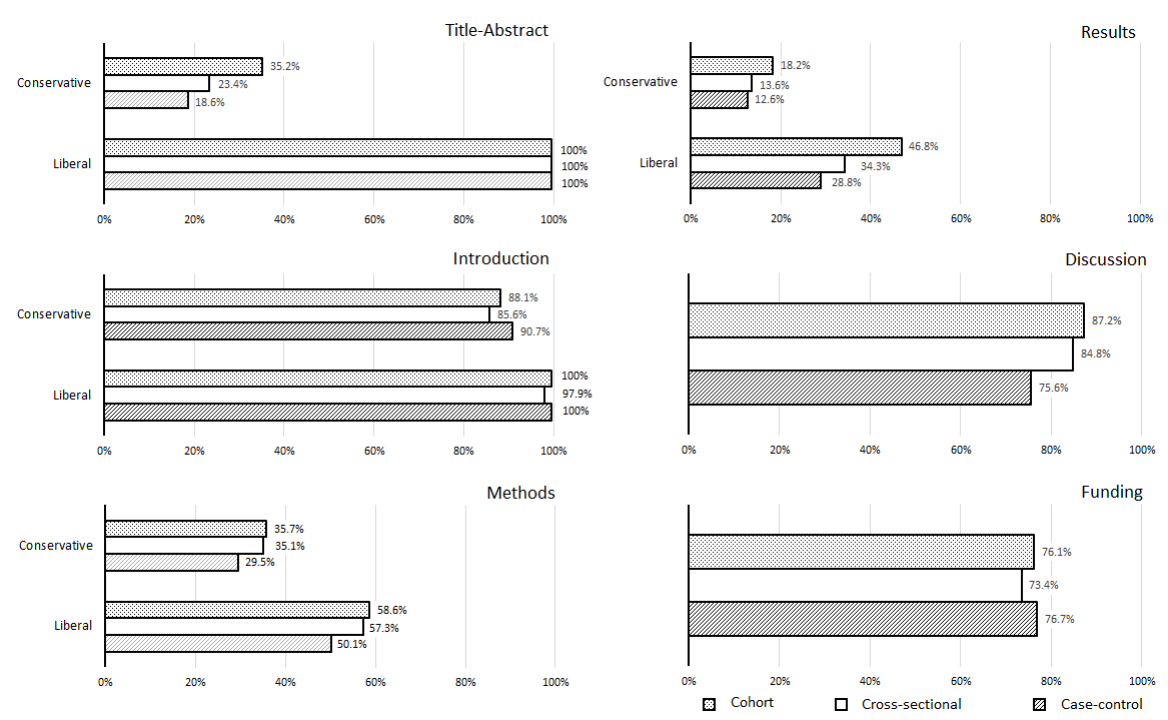

Fig 2. STROBE checklist response rates by paper section. Trends over the period 2009-2018 using a conservative or liberal scoring.

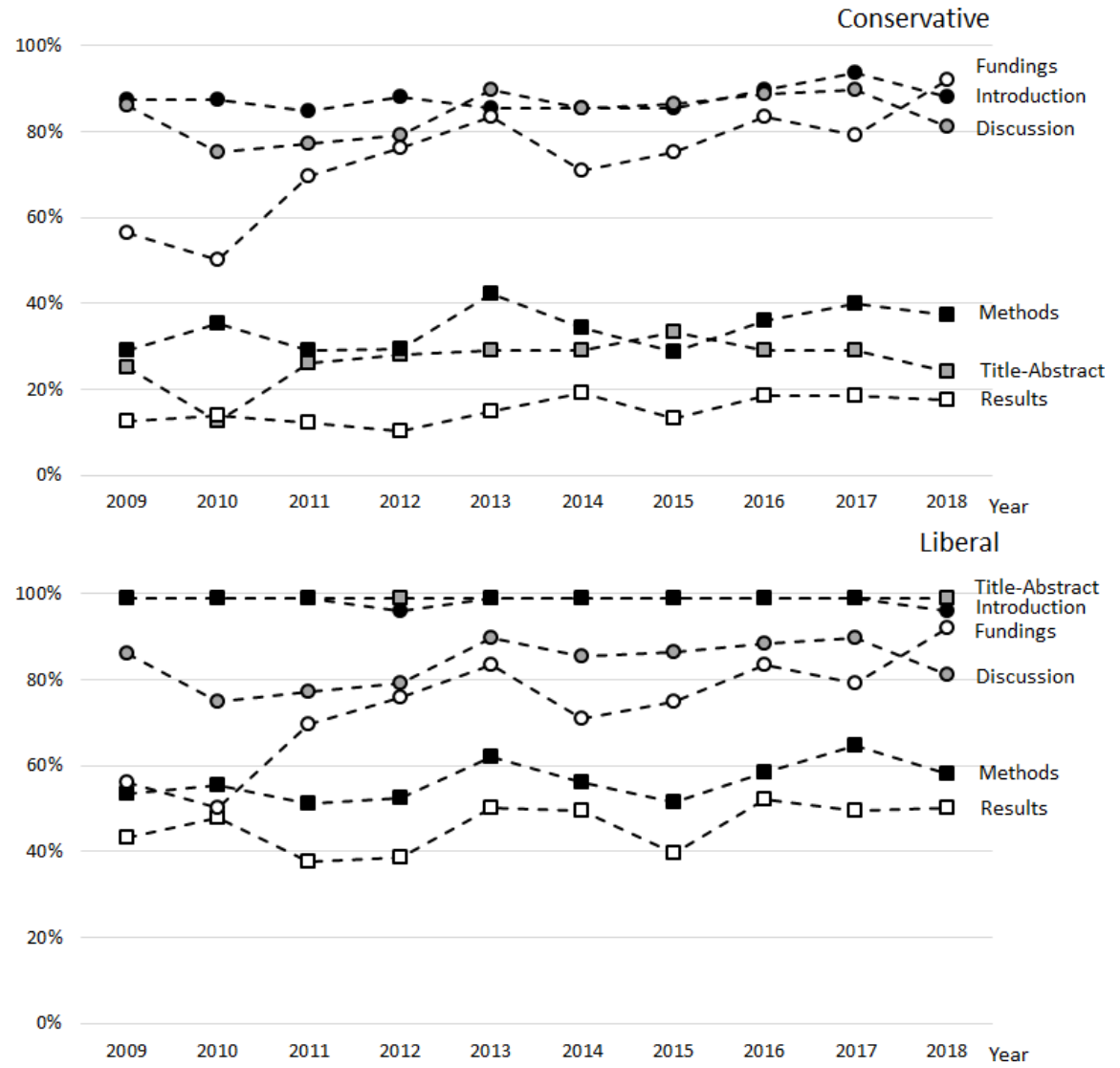

Fig 3. Multiple correspondence analyses of the STROBE checklist using a conservative or liberal scoring. 


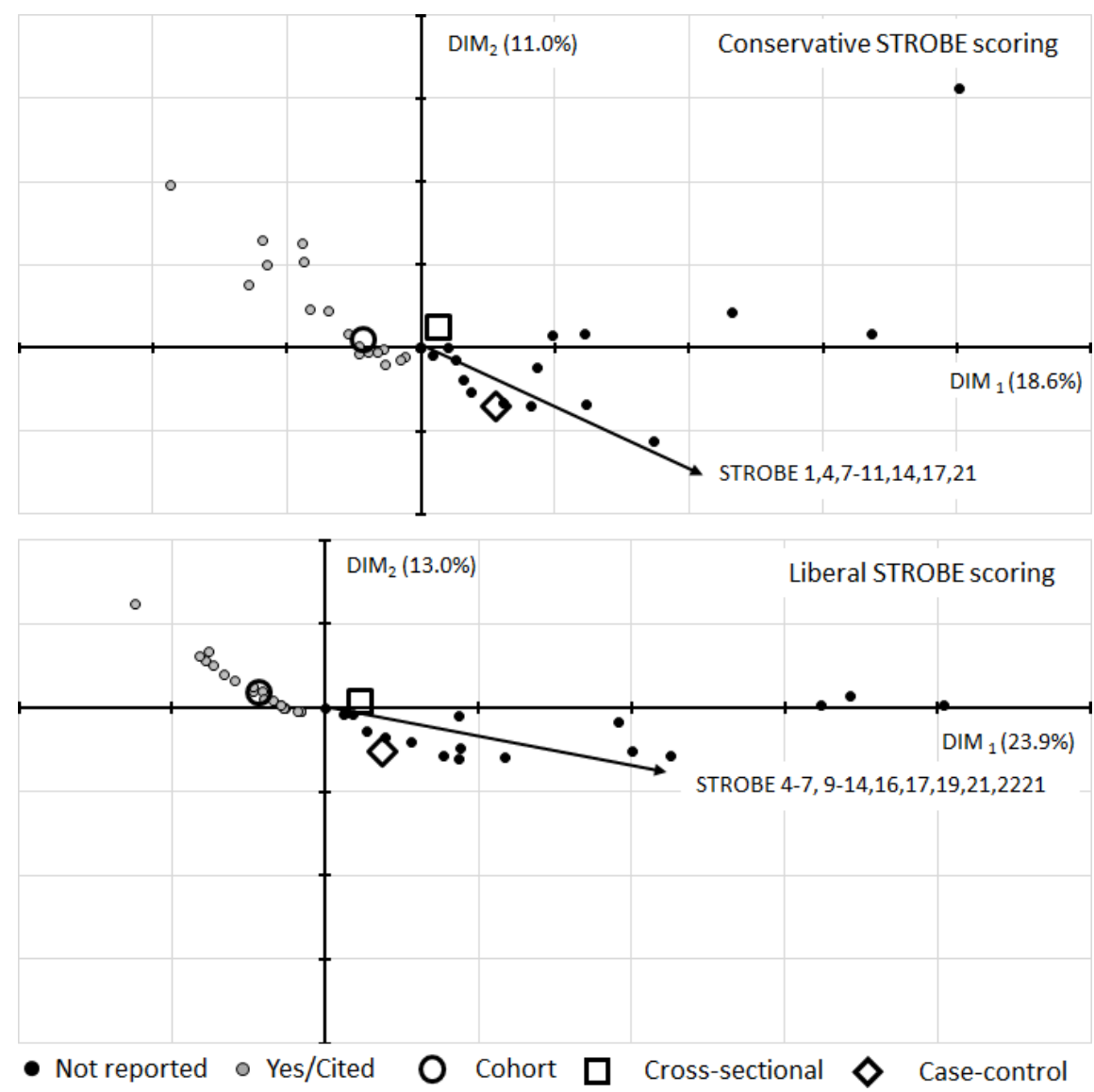

The 22 Strobe Items and their sub-items

Items are labelled as XX\# where XX represents the STROBE section [TA (title and abstract), IN (introduction), ME (methods), RE (results), DI (discussion)] and where \# represents the STROBE item [1-22]. The text is extracted from the STROBE explanatory article ${ }^{15}$ and the lowercase alphanumerical listing [(a), (b), (c), ...] indicates the sub-items.

- TA1 Title and abstract. (a) Indicate the study's design with a commonly used term in the title or the abstract. (b) Provide in the abstract an informative and balanced summary of what was done and what was found.

- IN2 Background/rationale. Explain the scientific background and rationale for the investigation being reported.

- IN3 Objectives. State specific objectives, including any prespecified hypotheses. (a) specific population, (b) exposures, (c) outcomes, (d) parameter to be estimated.

- ME4 Methods. Study design. Present key elements of study design early in the paper. Cohort: (a1) cohort, (a2) time period, (a3) exposure status; Case-control: (b1) case description, (b2) control description, (b3) source, (b4) population; Cross-sectional: (c1) population, (c2) timing.

- ME5 Setting. Describe the setting, locations, and relevant dates, including periods of recruitment, exposure, follow-up, and data collection.

- ME6 Participants. Cohort: (a1) eligibility criteria, (a2) selection sources, (a3) selection method, (a4) methods of follow-up Matched Cohort: (a5) For matched studies, give matching criteria and number of exposed and unexposed. Case-Controls: (b1) eligibility criteria, (b2) selection sources, (b3) selection method, (b4) rationale for the choice of cases and controls. Matched Case-control study: (b5) 
For matched studies, give matching criteria and the number of controls per case Cross-sectional (c1) eligibility criteria, (c2) selection sources, (c3) selection method

- ME7 Variables. Clearly define all (a) outcomes, (b) exposures and predictors, (c) potential confounders and effect modifiers. Give (d) diagnostic criteria, if applicable.

- ME8 Data sources/measurement. For each variable of interest, give (a) sources of data and (b) details of methods of assessment (measurement). Describe (c) comparability of assessment methods if there is more than one group.

- ME9 Bias. Describe any efforts to address potential sources of bias.

- ME10 Study size. Explain how the study size was arrived at.

- ME11 Quantitative variables. Explain how quantitative variables were (a) handled in the analyses. If applicable, describe (b) which groupings were chosen and why.

- ME12 Describe (a1) all statistical methods, including (a2) those used to control for confounding. (b) Describe any methods used to examine subgroups and interactions. (c) Explain how missing data were addressed. Cohort: (d1) If applicable, explain how loss to follow-up was addressed. Casecontrol: (d2) If applicable, explain how matching of cases and controls was addressed. Cross-sectional: (d3) If applicable, describe analytical methods taking account of sampling strategy. (e) Describe any sensitivity analyses.

- RE13 (a) Report numbers of individuals at each stage of study - eg numbers potentially eligible, examined for eligibility, confirmed eligible, included in the study, completing follow-up, and analysed. (b) Give reasons for non-participation at each stage. (c) Consider use of a flow diagram.

- RE14 Give characteristics of (a1) study participants (demographic, clinical, social) and information on (a2) exposures and (a3) potential confounders. (b) Indicate number of participants with missing data for each variable of interest. Cohort: (c) Summarise follow-up time (eg, average and total amount)

- RE15 Numbers Cohort: (a) Report numbers of outcome events or summary measures over time. Case-control: (b) Report numbers in each exposure category, or summary measures of exposure. Crosssectional: (c) Report numbers of outcome events or summary measures

- RE16 (a1) Give unadjusted estimates and, if applicable, (a2) confounder-adjusted estimates and (a3) their precision (eg, 95\% confidence interval). (a4) Make clear which confounders were adjusted for and why they were included. (b) Report category boundaries when continuous variables were categorized. (c) If relevant, consider translating estimates of relative risk into absolute risk for a meaningful time period.

- RE17 Other analyses. Report other analyses done - eg analyses of subgroups and interactions, and sensitivity analyses

- DI18 Discussion Key results. Summarise key results with reference to study objectives.

- DI19 Limitations. Discuss limitations of the study, taking into account sources of potential bias or imprecision. Discuss both direction and magnitude of any potential bias.

- DI20 Interpretation. Give a cautious overall interpretation of results considering objectives, limitations, multiplicity of analyses, results from similar studies, and other relevant evidence.

- DI21 Generalisability. Discuss the generalisability (external validity) of the study results.

- DI22 Funding. Give the source of funding and the role of the funders for the present study and, if applicable, for the original study on which the present article is based.

Supplementary table 1. Variables and rate of reporting where applicable

Items are labelled as XX\#_y where XX represents the STROBE section [TA (title and abstract), IN (introduction), ME (methods), RE (results), DI (discussion)], where \# represents the STROBE item [1-22], and where $\mathrm{y}$ is the lowercase alphanumerical listing $[(a),(b),(c), \ldots]$ indicating the sub-items defined in the section above.

\begin{tabular}{llllll}
\hline Item & Reporting & Item & Reporting & Item & Reporting \\
\hline TA1 & $28.3 \%$ & ME6_b3 & $56.9 \%$ & ME12_e & $22.0 \%$ \\
TA1_b & $91.0 \%$ & ME6_b4 & $91.2 \%$ & RE13_a & $47.5 \%$
\end{tabular}




\begin{tabular}{llllll}
\hline Item & Reporting & Item & Reporting & Item & Reporting \\
\hline IN2 & $50.0 \%$ & ME6_b5 & $26.3 \%$ & RE13_b & $27.8 \%$ \\
IN3_a & $94.2 \%$ & ME6_c1 & $82.5 \%$ & RE13_c & $17.5 \%$ \\
IN3_b & $92.8 \%$ & ME6_c2 & $38.6 \%$ & RE14_a1 & $84.8 \%$ \\
IN3_c & $99.1 \%$ & ME6_c3 & $14.0 \%$ & RE14_a2 & $91.0 \%$ \\
IN3_d & $88.8 \%$ & ME7_a & $90.6 \%$ & RE14_a3 & $46.6 \%$ \\
ME4_a1 & $50.0 \%$ & ME7_b & $85.7 \%$ & RE14_b & $27.4 \%$ \\
ME4_a2 & $93.0 \%$ & ME7_c & $37.2 \%$ & RE14_c & $57.9 \%$ \\
ME4_a3 & $93.0 \%$ & ME7_d & $58.3 \%$ & RE15_a & $96.5 \%$ \\
ME4_b1 & $50.0 \%$ & ME8_a & $94.2 \%$ & RE15_b & $96.5 \%$ \\
ME4_b2 & $94.7 \%$ & ME8_b & $98.7 \%$ & RE15_c & $98.2 \%$ \\
ME4_b3 & $80.7 \%$ & ME8_c & $31.4 \%$ & RE16_a1 & $90.6 \%$ \\
ME4_b4 & $96.5 \%$ & ME9 & $34.5 \%$ & RE16_a2 & $18.8 \%$ \\
ME4_c1 & $50.0 \%$ & ME10 & $42.2 \%$ & RE16_a3 & $44.4 \%$ \\
ME4_c2 & $78.9 \%$ & ME11_a & $31.4 \%$ & RE16_a4 & $52.9 \%$ \\
ME5 & $93.3 \%$ & ME11_b & $31.4 \%$ & RE16_b & $42.6 \%$ \\
ME6_a1 & $14.0 \%$ & ME12_a1 & $33.6 \%$ & RE16_c & $0.9 \%$ \\
ME6_a2 & $1.8 \%$ & ME12_a2 & $50.7 \%$ & RE17 & $72.2 \%$ \\
ME6_a3 & $10.5 \%$ & ME12_b & $57.4 \%$ & DI18 & $99.6 \%$ \\
ME6_a4 & $71.9 \%$ & ME12_c & $27.4 \%$ & DI19 & $84.3 \%$ \\
ME6_a5 & $8.8 \%$ & ME12_d1 & $24.6 \%$ & DI20 & $94.6 \%$ \\
ME6_b1 & $71.6 \%$ & ME12_d2 & $15.8 \%$ & DI21 & $60.5 \%$ \\
ME6_b2 & $97.2 \%$ & ME12_d3 & $35.8 \%$ & DI22 & $75.8 \%$ \\
\hline
\end{tabular}

\section{References}

1. Sánchez-Borges M, Martin BL, Muraro AM, et al. The importance of allergic disease in public health: an iCAALL statement. World Allergy Organ J . 2018;11(1):8. doi:10.1186/s40413-018-0187-2

2. Asher MI, Montefort S, Björkstén B, et al. Worldwide time trends in the prevalence of symptoms of asthma, allergic rhinoconjunctivitis, and eczema in childhood: ISAAC Phases One and Three repeat multicountry cross-sectional surveys. Lancet . 2006;368(9537):733-743. doi:10.1016/S0140-6736(06)69283-0

3. Björkstén B, Clayton T, Ellwood P, Stewart A, Strachan D, ISAAC Phase III Study Group. Worldwide time trends for symptoms of rhinitis and conjunctivitis: Phase III of the International Study of Asthma and Allergies in Childhood. Pediatr Allergy Immunol . 2008;19(2):110-124. doi:10.1111/j.1399-3038.2007.00601.x

4. Prescott SL, Pawankar R, Allen KJ, et al. A global survey of changing patterns of food allergy burden in children. World Allergy Organ J . 2013;6(1):21. doi:10.1186/1939-4551-6-21

5. Soriano JB, Abajobir AA, Abate KH, et al. Global, regional, and national deaths, prevalence, disabilityadjusted life years, and years lived with disability for chronic obstructive pulmonary disease and asthma, 1990-2015: a systematic analysis for the Global Burden of Disease Study 2015. The Lancet Respiratory Medicine . 2017;5(9):691-706. doi:10.1016/S2213-2600(17)30293-X

6. Genuneit J, Seibold AM, Apfelbacher CJ, et al. Overview of systematic reviews in allergy epidemiology. Allergy . 2017;72(6):849-856. doi:10.1111/all.13123

7. Genuneit J, Seibold AM, Apfelbacher CJ, et al. The state of asthma epidemiology: an overview of systematic reviews and their quality. Clin Transl Allergy . 2017;7:12. doi:10.1186/s13601-017-0146-y

8. Ryan D, Levy M, Morris A, Sheikh A, Walker S. Management of allergic problems in primary care: time for a rethink? Prim Care Respir J . 2005;14(4):195-203. doi:10.1016/j.pcrj.2005.01.003 
9. Jutel M, Angier L, Palkonen S, et al. Improving allergy management in the primary care network-a holistic approach. Allergy . 2013;68(11):1362-1369.

10. Neta G, Glasgow RE, Carpenter CR, et al. A Framework for Enhancing the Value of Research for Dissemination and Implementation. Am J Public Health . 2015;105(1):49-57. doi:10.2105/AJPH.2014.302206

11. Brownson RC, Colditz GA, Proctor EK. Dissemination and Implementation Research in Health: Translating Science to Practice. Oxford University Press; 2017.

12. Simera I, Altman DG, Moher D, Schulz KF, Hoey J. Guidelines for reporting health research: the EQUATOR network's survey of guideline authors. PLoS Med . 2008;5(6):e139.

13. von Elm E, Altman DG, Egger M, et al. The Strengthening the Reporting of Observational Studies in Epidemiology (STROBE) statement: guidelines for reporting observational studies. Lancet . 2007;370(9596):1453-1457. doi:10.1016/S0140-6736(07)61602-X

14. da Costa BR, Cevallos M, Altman DG, Rutjes AWS, Egger M. Uses and misuses of the STROBE statement: bibliographic study. BMJ Open . 2011;1(1):e000048. doi:10.1136/bmjopen-2010-000048

15. Vandenbroucke JP, von Elm E, Altman DG, et al. Strengthening the Reporting of Observational Studies in Epidemiology (STROBE): explanation and elaboration. Ann Intern Med . 2007;147(8):W163-194. doi:10.7326/0003-4819-147-8-200710160-00010-w1

16. Pouwels KB, Widyakusuma NN, Groenwold RHH, Hak E. Quality of reporting of confounding remained suboptimal after the STROBE guideline.J Clin Epidemiol . 2016;69:217-224. doi:10.1016/j.jclinepi.2015.08.009

17. Aghazadeh-Attari J, Mobaraki K, Ahmadzadeh J, Mansorian B, Mohebbi I. Quality of observational studies in prestigious journals of occupational medicine and health based on Strengthening the Reporting of Observational Studies in Epidemiology (STROBE) Statement: a cross-sectional study. BMC Res Notes . 2018;11(1):266. doi:10.1186/s13104-018-3367-9

18. Hendriksma M, Joosten MHMA, Peters JPM, Grolman W, Stegeman I. Evaluation of the Quality of Reporting of Observational Studies in Otorhinolaryngology - Based on the STROBE Statement. PLoS ONE . 2017;12(1):e0169316. doi:10.1371/journal.pone.0169316

19. Mannocci A, Saulle R, Colamesta V, et al. What is the impact of reporting guidelines on Public Health journals in Europe? The case of STROBE, CONSORT and PRISMA. Journal of Public Health . 2014;37(4):737-740. doi:10.1093/pubmed/fdu108

20. Carneiro CFD, Queiroz VGS, Moulin TC, et al. Comparing quality of reporting between preprints and peer-reviewed articles in the biomedical literature. Res Integr Peer Rev . 2020;5(1):16. doi:10.1186/s41073020-00101-3

21. Rahmani N, Salehi A, Molavi Vardanjani H, Marzban M, Behbood A. Using STROBE checklist to assess the reporting quality of observational studies affiliated with Shiraz University of Medical Sciences, and its correlates: a scientometric study from Iran. Scientometrics . 2020;122(2):989-1001. doi:10.1007/s11192-019$03317-3$

22. Aschengrau A, Seage GR. Essentials of Epidemiology in Public Health . Jones \& Bartlett Publishers; 2013.

23. Sedgwick P. Estimating the population at risk. Bmj . 2012;345:e6859.

24. Kopec JA, Esdaile JM. Bias in case-control studies. A review.J Epidemiol Community Health . 1990;44(3):179-186. doi:10.1136/jech.44.3.179

25. Moher D, Altman DG. Four Proposals to Help Improve the Medical Research Literature. PLOS Medicine . 2015;12(9):e1001864. doi:10.1371/journal.pmed.1001864 
26. Agha RA, Fowler AJ, Limb C, et al. Impact of the mandatory implementation of reporting guidelines on reporting quality in a surgical journal: A before and after study. International Journal of Surgery . 2016;30:169-172. doi:10.1016/j.ijsu.2016.04.032

27. Blanco D, Altman D, Moher D, Boutron I, Kirkham JJ, Cobo E. Scoping review on interventions to improve adherence to reporting guidelines in health research. BMJ Open . 2019;9(5):e026589. doi:10.1136/bmjopen2018-026589 